\title{
Avaliação global do idoso através da Telemedicina frente a pandemia do COVID-19: um relato de experiência
}

\author{
Global assessment of the elderly through Telemedicine in the face of the COVID-19 \\ pandemic: an experience report
}

Evaluación global del adulto mayor a través de la Telemedicina ante la pandemia COVID-

19: un informe de experiencia

Isabella Junho dos Santos Souza ${ }^{1 *}$, Rafaela Rezende Celani ${ }^{1}$, Domitila Natividade Figueiredo Lopes $^{1}$, Renata Licursi Nogueira ${ }^{1}$.

\section{RESUMO}

Objetivo: Compreender a avaliação global do idoso, realizada de maneira remota por meio de plataformas online, durante o período de isolamento social ocasionado pela pandemia do COVID-19. Relato de experiência: Trata-se de um estudo descritivo acerca de uma experiência vivenciada por estudantes de Medicina em parceria com centros de cuidado diário direcionados aos idosos. Mediante a aplicação de formulários online, avaliou-se a saúde orgânica, social e psicológica de pacientes com idade superior a 60 anos. Uma vez que o processo de senescência é acompanhado de um declínio da capacidade funcional, a população idosa torna-se mais exposta as condições de vulnerabilidade. Dessa maneira, visando a promoção da continuidade do cuidado integrado, fez-se necessário adequar os atendimentos para a forma remota a fim de não expor tal grupo a riscos ante a pandemia. Considerações finais: A experiência visou a manutenção da qualidade de vida e atenção à saúde do idoso através da Telemedicina, uma ferramenta promissora no âmbito social e na relação médico-paciente, sobretudo nos tempos atuais referentes a pandemia acarretada pela doença COVID-19.

Palavras-chave: COVID-19, Envelhecimento, Telemedicina.

\begin{abstract}
Objective: To understand the global assessment of the elderly, carried out remotely through online platforms, during the period of social isolation caused by the COVID-19 pandemic. Experience report: This is a descriptive study about an experience lived by medical students in partnership with daily care centers aimed at the elderly. Through the application of online forms, the organic, social and psychological health of patients aged over 60 years was evaluated. Once the senescence process is accompanied by a decline in functional capacity, the elderly population becomes more exposed to conditions of vulnerability. Thus, aiming to promote the continuity of integrated care, it was necessary to adapt the care to the remote form in order not to expose this group to risks in the face of the pandemic. Final considerations: The experience aimed to maintain the quality of life and health care of the elderly through Telemedicine, a promising tool in the social sphere and in the doctor-patient relationship, especially in current times regarding the pandemic caused by the disease COVID-19.
\end{abstract}

Keywords: COVID-19, Aging, Telemedicine.

1 Universidade de Franca (UNIFRAN), Franca - SP. *E-mail: isabella-junho@hotmail.com 


\section{RESUMEN}

Objetivo: Comprende una evaluación global de las personas mayores, realizada de forma remota a través de plataformas en línea, durante el período de aislamiento social provocado por la pandemia COVID-19. Informe de experiencia: Se trata de un estudio descriptivo sobre una experiencia vivida por estudiantes de medicina en alianza con centros de atención diaria dirigidos a personas mayores. Mediante la aplicación de formularios online se evaluó la salud orgánica, social y psicológica de los pacientes mayores de 60 años. Una vez que el proceso de senescencia se acompaña de una disminución de la capacidad funcional, una población anciana se vuelve más expuesta como condiciones de vulnerabilidad. Así, además de promover la continuidad de la atención integral, era necesario brindar una atención adecuada a la forma remota para no exponer a este grupo a riesgos antes de la pandemia. Consideraciones finales: La experiencia tuvo como objetivo mantener la calidad de vida y la atención de la salud de las personas mayores a través de la Telemedicina, una herramienta prometedora en el ámbito social y en la relación médico-paciente, especialmente en los tiempos actuales con respecto a la pandemia provocada por la enfermedad COVID-19.

Palabras clave: COVID-19, Envejecimiento, Telemedicina.

\section{INTRODUÇÃO}

Define-se como idoso, segundo a Organização Mundial de Saúde (OMS), todo indivíduo com 60 anos ou mais. De acordo com dados do IBGE de 2018, com respaldo no Censo Demográfico de 2010, o Brasil possuía mais de 28 milhões de pessoas dentro dessa idade, com uma representação de cerca de $13 \%$ da população total, sendo em sua maioria composta por mulheres. Além disso, estima-se que essa taxa tem a tendência em dobrar nas décadas seguintes (PERISSE C e MARLI M, 2019).

Esse fato pode ser explicado em decorrência da diminuição nas taxas de fecundidade e natalidade, como também melhorias e amplificação do cuidado em saúde, que proporcionam um aumento na qualidade de vida da população, trazendo como consequência um respectivo crescimento no tempo vivido (TAVARES RE, et. al., 2017).

Sendo assim, observa-se um processo de envelhecimento crescente, o qual embora não seja sinônimo de adoecimento, é acompanhado de uma maior prevalência de acometimentos funcionais e orgânicos, relacionados a perda da autonomia e independência. Como resultado, tem-se nessa faixa etária, uma acentuada procura de atendimento na área da saúde, necessitando de assistência e atenção contínuas, a fim de proporcionar um envelhecer digno e saudável (VERAS RP e OLIVEIRA M, 2018).

Diante dessa fragilidade presente em tal grupo populacional, aliada ao contexto da atual pandemia do COVID-19, ocasionada pelo vírus SARS-CoV-2 e com início entre os meses de fevereiro e março do ano de 2020, viu-se necessário a criação de medidas protetivas com o intuito de expandir a continuidade ao cuidado e a redução do risco de contágio e disseminação desse vírus (BARBOSA ACS, et al., 2020). É nesse cenário que a Telemedicina, uma ferramenta já antes utilizada, ganha destaque e aprimoramento no âmbito da saúde ao idoso (DIAS SCF, et al., 2018).

Nessas circunstâncias, a Telemedicina torna-se fundamental para a promoção, prevenção e readequação dos cuidados em saúde, proporcionando um auxílio médico remoto e reduzindo os riscos aos pacientes, familiares e profissionais da saúde. O instrumento em questão pode ser entendido como um efeito dos aprimoramentos em tecnologia, que beneficiam de forma positiva as variadas esferas da sociedade, com ênfase na saúde (BRITO BO e LEITAO LPC, 2020).

Dessa forma, a telemedicina é entendida como um recurso de comunicação online com a finalidade de propagar informações e conhecimentos pessoais e da área médica. Conclui-se para tal que, embora o contato médico-paciente seja um dos pilares para o estabelecimento de um vínculo humanizado e individualizado, os atendimentos remotos são essenciais frente a situações em que a distância é um fator crítico, como em meio a pandemia atual (KIELING D, et al., 2021). 
Tendo em vista o crescimento das teleconsultas, o Conselho Federal de Medicina em conjunto com o Poder Legislativo, decretou a lei № 13.989 , em 15 de abril de 2020, com o intuito de regulamentar e autorizar o uso da Telemedicina durante a pandemia causada pelo SARS-CoV-2, ficando no dever médico o papel de expor ao paciente as restrições advindas desse método, tal como a impossibilidade de obter um contato direto e um exame físico completo. Essa lei em questão reforça o princípio de integralidade presente no Sistema Único de Saúde, objetivando atender as necessidades individuais da população idosa (BRASIL, 2020).

Os profissionais de saúde ao exercerem as teleconsultas, no contexto do sistema de saúde, devem ser aptos e habilitados a reconhecerem àqueles com maior deterioração funcional, objetivando proporcionar não apenas maior longevidade, como também maior bem-estar e comodidade (MANSO MEG, et al., 2018). Por conseguinte, a denominada Avaliação Geriátrica Ampla (AGA) faz-se como um excelente meio de análise das diversas esferas biopsicossociais do idoso, como a função cognitiva, a locomoção, o estado mental, a funcionalidade, entre outros, avaliando o indivíduo como um todo. Aliado a esse atributo, a AGA destaca-se por propiciar um acompanhamento comparativo do paciente a longo prazo, observando se há boa adesão terapêutica e evoluções no estado clínico (SARAIVA LB, et al., 2017).

Para análise dessas diversas categorias, enquadram-se testes descritivos que permitem a elaboração de uma assistência individualizada e multidimensional, garantindo aos idosos uma participação ativa durante toda a consulta remota (MARQUES GCS, et al., 2018).

Para tanto, tem-se como exemplo para uma investigação cognitiva, objetiva e de simples execução, o Mini Exame do Estado Mental (MEEM), composto por questionamentos com pontuações no que se refere a orientação, linguagem, cálculo, orientação no tempo e espaço, memorização e domínio visual. Já para a verificação da funcionalidade, pode-se lançar mão da Escala de Katz, que avalia as Atividades Básicas de Vida Diária (ABVD), como vestir-se, banhar-se, alimentar-se, realização de higiene pessoal e controle dos esfíncteres, fatores esses que são determinantes da autonomia do idoso. Por fim, tem-se a Escala de Depressão Geriátrica (EDG), que é aplicada para rastreamento do estado depressivo, sendo constituída por perguntas acerca da condição emocional e afetiva (ALIXANDRE AL, et al., 2019).

Ante ao exposto, considera-se o idoso como um ser dotado de individualidades que demandam uma atenção diferenciada, sobretudo em tempos de pandemia. Logo, o vigente trabalho teve como objetivo compreender e avaliar o atendimento da pessoa idosa de forma global, através de plataformas online por videoconferência e sua correlação com a pandemia ocasionada pelo COVID-19, a fim de promover a manutenção de um cuidado íntegro e singular à saúde desse grupo populacional. Os meios de comunicação, embora ainda não sejam totalmente difundidos e estejam em fases de aprimoramentos prospectivos, ganharam espaço e destaque imprescindíveis ao setor de saúde pública, notadamente nos cuidados inerentes ao processo do envelhecimento.

\section{RELATO DE EXPERIÊNCIA}

O presente trabalho foi realizado através do Programa de Integração do Ensino/Saúde da Família (PIESF), disciplina essa constituinte da grade curricular de uma Universidade do interior paulista, do primeiro ao quarto ano do curso de Medicina. Tal módulo visa viabilizar aos estudantes um contato com a Rede Básica de Saúde, ao integrá-los a centros de assistência médica, como as Unidades Básicas de Saúde (UBS). Como resultado, assegura-se aos acadêmicos a correlação da teoria aprendida com a prática da mesma, englobando diversos ramos do conhecimento, com ênfase, nesse estudo, na saúde do idoso. É estabelecido, dessa maneira, um aprendizado concreto, otimizado e eficaz.

A atividade foi desenvolvida por duas alunas do terceiro ano (sexto período) sob orientação de um docente responsável, o qual efetuou a intermediação para que consultas fossem realizadas em um Centro Dia localizado no interior do estado de São Paulo (SP). Esse trata-se de uma unidade pública cujo propósito é oferecer aos idosos acolhimento e reintegração social, sobretudo nos contextos em que a família não possui requisitos para o cuidado integral desse público-alvo. Nesses locais, busca-se prover ao idoso incentivos ao cuidado pessoal, a manutenção da autonomia e independência, ao fortalecimento dos vínculos familiares e 
as atividades socioeducativas, sendo as ações orientadas de acordo com o perfil individual de cada usuário, conforme suas necessidades específicas.

Em razão da pandemia pelo COVID-19, as consultas presenciais estavam impossibilitadas nesse Centro de Referência ao idoso. Logo, foram primordiais para o atendimento ferramentas de comunicação eletrônicas do Google, como o Forms, usado como questionário guia, e o Meet, aplicativo de videoconferência. O primeiro abrangia questões referentes aos pacientes, como nome, idade, moradia, uso de medicações, comorbidades e estado nutricional, englobando aspectos da identificação e sua história prévia e atual. O segundo, por sua vez, instaurou um contato mais próximo e reservado para a concretização da atividade proposta.

Os pacientes entrevistados frequentavam o Centro Dia e, com o auxílio de funcionários do local, as perguntas eram repassadas aos idosos. De acordo com os questionários aplicados durante a identificação, observou-se que as doenças mais prevalentes eram Hipertensão Arterial Sistêmica (HAS) e Diabetes Mellitus tipo 2 (DM 2). Não foi possível realizar o exame físico, uma limitação referente às teleconsultas, sendo levado em conta medidas anteriores da Pressão Arterial, peso e glicemia em jejum. Esses dados foram obtidos através de fichas cadastrais presentes no local, os quais apresentaram relevância para a compreensão do processo saúde-doença e para possíveis intervenções benéficas ao público-alvo desenvolvidas pelas alunas.

Para a Avaliação Geriátrica Ampla (AGA), aplicaram-se diversas escalas, sendo essas o Mini Exame do Estado Mental (MEEM), empregado para análise da função cognitiva; a Escala de Katz e a Escala de Lawton/Brody, utilizadas na investigação da funcionalidade por meio de Atividades Básicas de Vida Diária (ABVD) e Atividades Instrumentais de Vida Diária (AIVD), respectivamente; a Escala de Depressão Geriátrica (EDG), que auxilia no reconhecimento de possíveis sintomas depressivos, e, por fim, a Escala de Eficácia de Quedas Internacional (FES-I), identificando o nível de preocupação de quedas durante a realização de atividades diárias, como ao caminhar, ao subir escadas e preparar refeições, entre outras.

Como resultado dessa experiência, as alunas conseguiram reconhecer as individualidades de cada idoso e, em decorrência disso, entender que o processo de envelher necessita de um olhar minuncioso e individual, sobretudo no contexto em que a Telemedicina tornou-se um recurso fundamental, na tentativa de promover uma assistência contínua nos tempos de pandemia. Por conseguinte, a presença de redes adequadas de apoio foi considerada como um dos pilares fundamentais para a manutenção da estabilidade física e emocional dos idosos, não somente durante esse período, mas também em todo o curso da senescência.

\section{DISCUSSÃO}

O processo de envelhecimento atual é caracterizado por ser um fenômeno complexo e singular, que engloba variadas perspectivas intrínsecas e extrínsecas ao indivíduo, tais como o padrão de vida, circunstâncias sociais, mentais, econômicas e interpessoais, aliadas a uma possível incapacidade funcional, com maior prevalência de doenças agudas e crônicas (SILVA FLC, et al., 2019). Nesse cenário, o Envelhecimento Ativo, proposto pela Organização Mundial de Saúde (OMS), ganha destaque, tornando-se necessárias medidas para a sua aplicabilidade. Esse pode ser atribuído ao aprimoramento das suas três bases principais: segurança, saúde e participação, a fim de propiciar não apenas um aumento na expectativa de vida, como também uma evolução na qualidade da mesma, assegurando ao idoso uma atuação contínua e diligente nos diversos setores da sociedade (SCIAMA DS, et al., 2020).

Frente a isso, a promoção ao autocuidado, independência, autonomia e socialização intitula-se como primordial para a manutenção de uma vida regularmente ativa e um envelhecer dotado de hábitos saudáveis, dignidade e bem-estar. No que diz respeito às responsabilidades a cargo da saúde pública pode-se ressaltar a importância da implementação de políticas governamentais direcionadas ao grupo populacional em destaque, com a finalidade de garantir que seus direitos sejam assegurados e respeitados (SOUSA NFS, et al., 2018).

A Política Nacional do Idoso, sancionada pela lei № 8.842, de 4 de janeiro de 1994, aliada a Política Nacional de Saúde da Pessoa Idosa, decretada pela Portaria № 2.528 de 19 de outubro de 2006 promoveram a regulamentação de ações voltadas não somente na área da saúde, como também na educação, trabalho e 
previdência social, justiça, cultura, esporte e lazer, transformando o papel do idoso em uma forma ativa no que tange aos seus direitos e deveres (MINISTÉRIO DA SAÚDE, 2006). O Centro-Dia, local em que os idosos do presente estudo frequentavam, constitui-se como um dos recursos de atendimento implementados pela Política Nacional do Idoso, sendo caracterizado por ser um espaço designado a estadia e suporte diurnos. Tal assistência engloba a manutenção das habilidades referentes a atividades básicas da vida diária (ABVD) e apoio multidisciplinar, como de psicólogos e fisioterapeutas (BONATELLI LCS, et al., 2018).

Mediante a tal contexto, a Avaliação Global do Idoso qualifica-se como um meio crucial ao atendimento geriátrico, que pode ser aplicado em locais de suporte aos idosos, com ênfase no reconhecimento de Doenças Crônicas Não Transmissíveis (DCNT), altamente prevalentes nesse grupo populacional e que se associam à deterioração fisiológica, inerente ao processo de senescência. Dentre as principais DCNT, salienta-se o Diabetes Mellitus tipo 2 (DM 2), neoplasias malignas, doenças respiratórias e doenças cardiovasculares, como a Hipertensão Arterial Sistêmica (HAS) (SCARAVONAT G, et al., 2019). Essas são caracterizadas por apresentarem etiologia não infecciosa e curso prolongado, sendo necessário terapia medicamentosa contínua para controle e manutenção.

Como observado no referido estudo, os idosos que foram avaliados nas teleconsultas, apresentavam como principais comorbidades a HAS e 0 DM 2, que estão entre os distúrbios de maior índice de morbimortalidalidade. A HAS representa uma condição em que os níveis pressóricos permanecem aumentados de forma contínua ( $\geq 140 \times 90 \mathrm{mmHg}$ ), com repercussões nos principais órgãos-alvo (coração, rins e encéfalo) (SILVA FHM, et al., 2019). Em decorrência da alta prevalência da HAS, foi orientada a manutenção de hábitos de vida saudáveis, como alimentação balanceada e prática regular de exercícios físicos, como também, a procura de auxílio médico para avaliar possíveis necessidades de alterações no plano terapêutico farmacológico e não farmacológico.

Já o DM 2, forma mais comum de apresentação, é identificado como um quadro de hiperglicemia (glicemia em jejum $\geq 126 \mathrm{mg} / \mathrm{dl}$ ) resultante tanto de um estado de resistência à insulina como de uma menor produção desta. Dentre os indivíduos predispostos, ressalta-se os obesos, os não praticantes de atividade física e/ou aqueles com histórico familiar positivo (BERTONHI LG, DIAS JCR, 2018). Correlatando aos idosos entrevistados que apresentavam essa comorbidade, aconselhou-se a manutenção de consultas de rotina com o médico responsável pelo acompanhamento de tal patologia. Quanto as medidas não farmacológicas, foi instruído, assim como na HAS, a continuidade de um estilo de vida balanceado, incluindo uma alimentação equilibrada com uma ingesta hídrica apropriada, atividades físicas frequentes, realização de atividades de interesse pessoal como manutenção do lazer, noites de sono regulares e acompanhamentos periódicos em serviços de saúde.

Além dos pontos já abordados, a autopercepção do idoso frente a si mesmo constitui-se como um complemento na avaliação integral do paciente geriátrico, em razão de abranger concepções acerca de sua qualidade de vida, caracterizando-se como um preditor de saúde, bem-estar e morbimortalidade (VAZ CT, et al., 2020). Tal fato se confirma no reconhecimento de maiores índices de mortalidade observados nos casos em que a autoanálise é desfavorável, inferindo que tanto aspectos cognitivos quanto emocionais também interferem no processo saúde-doença (LEAL RC, et al., 2020). Em paralelo ao atendimento realizado nesse estudo, a autoavaliação, interpretada pelas alunas, foi vista como positiva, dado que os idosos apresentavam conhecimentos e perspectivas otimistas a respeito de suas condições clínicas.

Por fim, a experiência ilustrada foi realizada de maneira satisfatória, contribuindo para o aprendizado das alunas ao integrar a aplicação da teoria com a prática e ao reconhecer a Avaliação Geriátrica Ampla, ambos viabilizados através de uma comunicação assertiva com os pacientes. Por sua vez, os idosos beneficiaramse acerca do estudo, que visava a promoção e prevenção da saúde, além de poder, por meio de uma atividade social possibilitada pela Telemedicina, expor suas ideias, angústias e expectativas sobre suas vertentes biopsicossociais, mesmo em um período de isolamento devido a pandemia. Portanto, a população geriátrica, que ganha cada vez mais espaço no cenário mundial, requer uma atenção mais cuidadosa e direcionada, de forma a permitir, não somente um aumento na expectativa de vida, como também um progresso associado a melhorias na qualidade da mesma. 


\section{REFERÊNCIAS}

1. PERISSE C e MARLI M. Agência IBGE Notícias. 2019. Disponível em: https://agenciadenoticias.ibge.gov.br/agencianoticias/2012-agencia-de-noticias/noticias/24036-idosos-indicam-caminhos-para-uma-melhor-idade. Acesso em: 10 de agosto de 2021.

2. TAVARES RE, et. al. Envelhecimento saudável na perspectiva de idosos: uma revisão integrativa. Revista Brasileira de Geriatria e Gerontologia, 2017; 20(6): p. 889-900.

3. VERAS RP e OLIVEIRA M. Envelhecer no Brasil: a construção de um modelo de cuidado. Ciência e Saúde Coletiva. 2018; 23(6).

4. BARBOSA ACS, et al. Atenção ao idoso frente à pandemia por Covid-19. Revista de Saúde Pública do Paraná. 2020; 3(1).

5. DIAS SCF, et al. Telemedicina Aplicada aos Cuidados dos Idosos no Brasil. In: Anais do V Congresso Brasileiro de Eletromiografia e Cinesiologia e X Simpósio de Engenharia Biomédica; 2018; Uberlândia. p. 843-846.

6. BRITO BO e LEITAO LPC. Telemedicina no Brasil: Uma estratégia possível para o cuidado em saúde em tempo de pandemia? Revista Saúde em Redes. 2020; 6(2).

7. KIELING D, et al. A importância da telemedicina no contexto da pandemia de COVID-19. Fag Journal of Healthy (FJH). $2021 ; 3(1)$.

8. BRASIL. Gov.br. 2020. Disponível em: https://www.in.gov.br/en/web/dou/-/lei-n-13.989-de-15-de-abril-de-2020252726328. Acesso em: 9 de agosto de 2021.

MANSO MEG, et al. Avaliação Multidimensional do Idoso: resultados em um grupo de indivíduosvinculados a uma operadora de planos de saúde. Revista Kairós Gerontologia. 2018; 21(1).

9. SARAIVA LB, et al. Avaliação Geriátrica Ampla e sua Utilização no Cuidado de Enfermagem a Pessoas Idosas. Journal of Health Sciences. 2017; 19(4).

10. MARQUES GCS, et al. Profissional Enfermeiro: Competências e habilidades para a avaliação multidimensional da pessoa idosa. Revista Kairós Gerontologia. 2018; 21(2).

11. ALIXANDRE AL, et al. Avaliação Multidimensional como Instrumento Complementar para a Consulta de Enfermagem à Pessoa Idosa na Atenção Básica. In VI Congresso Internacional de Envelhecimento Humano; 2019; Campina Grande. p. 1-12.

12. SILVA FLC, et al. Envelhecimento ativo: o papel da fisioterapia na melhoria da qualidade de vida da pessoa idosa: revisão integrativa. Revista Uningá. 2019; 56(4).

13. SCIAMA DS, et al. Envelhecimento ativo: representações sociais dos profissionais de saúde das Unidades de Referência à Saúde do Idoso. Revista da Escola de Enfermagem da USP. 2020; 54(e03605).

14. SOUSA NFS, et al. Envelhecimento ativo: prevalência e diferenças de gênero e idade em estudo de base populacional. Cadernos de Saúde Pública. 2018; 34(11).

15. MINISTÉRIO DA SAÚDE. Portaria № 2.528 de 19 de outubro de 2006, Aprova a Política Nacional de Saúde da Pessoa Idosa. Brasil, 2006. Disponível em: https://bvsms.saude.gov.br/bvs/saudelegis/gm/2006/prt2528_19_10_2006.html. Acesso em: 11 de novembro de 2021.

16. BONATELLI LCS, et al. Centro-dia: uma opção no atendimento da pessoa envelhecente com deficiência intelectual. Saúde em Debate. 2018; 42(118).

17. SCARAVONAT G, et al. Avaliação Geriátrica Ampla de Idosos de uma Universidade Pública da Maturidade: Vivências de Acadêmicas de Nutrição. Revista Humanidades e Inovação. 2019; 6(11).

18. SILVA FHM, et al. Intervenção de Saúde sobre Hipertensão e Diabetes. Revista de Enfermagem UFPE Online. 2019; 13(e240593).

19. BERTONHI LG, DIAS JCR. Diabetes mellitus tipo 2: aspectos clínicos, tratamento e conduta dietoterápica. Ciências Nutricionais Online. 2018; 2(2).

20. VAZ CT, et al. Fatores associados à autopercepção de saúde entre idosos de grupos comunitários. Revista Brasileira em Promoção da Saúde. 2020; 33(10328).

21. LEAL RC, et al. Percepção de saúde e comorbidades do idoso: perspectivas para o cuidado de. Brazilian Journal of Development. 2020; 6(7). 